
\title{
25 Research Soure \\ Significance of time from injury to surgery in the functional recovery of individuals with traumatic acute subdural hematoma
}

Shih-Han Chen ( $\nabla$ shihan1963@gmail.com )

Ditmanson Medical Foundation Chia-Yi Christian Hospital https://orcid.org/0000-0002-7584-9346 Jui-Ming Sun

Ditmanson Medical Foundation Chia-Yi Christian Hospital

Wen-Kuei Fang

Ditmanson Medical Foundation Chia-Yi Christian Hospital

Research article

Keywords: Time from injury to surgery, Traumatic acute subdural hematoma, Surgical outcomes of TASDH, ROC curve

Posted Date: December 17th, 2019

DOI: https://doi.org/10.21203/rs.2.19040/v1

License: (c) (i) This work is licensed under a Creative Commons Attribution 4.0 International License. Read Full License 


\section{Abstract}

Background: The time from injury to surgery (TIS) is critical in the functional recovery of individuals with traumatic acute subdural hematoma (TASDH). However, only few studies have confirmed such notion.

Methods: The data of TASDH patients who were surgically treated in Chia-Yi Christian Hospital between January 2008 and December 2015 were collected. The significance of variables, including age, sex, traumatic mechanism, coma scale, midline shift on brain computed tomography (CT) scan, and TIS, in functional recovery was assessed using the student's $\mathrm{t}$-test, chi-square test, univariate and multivariate models, and receiver operating characteristic (ROC) curve.

Results: A total of 37 patients achieved functional recovery (outcome scale score of 4 or 5 ) and 33 patients had poor recovery (outcome scale score of 1-3) after at least 1 year of follow-up. No significant difference was observed in terms of age, sex, coma scale score, traumatic mechanism, or midline shift on brain CT scan between the functional and poor recovery groups. TIS was found to be significantly shorter in the functional recovery group than in the poor recovery group ( $145.5 \pm 27.0$ vs. $181.9 \pm 54.5$, Pvalue $=0.001)$. TIS and midline shift $\geq 10 \mathrm{~mm}$ were significant factors for functional outcomes in the multivariate regression models. The analysis of TIS with the ROC curve between these two groups showed that the threshold time for functional recovery in comatose patients and those with TASDH who were surgically treated was 2 hours and 57.5 minutes.

Conclusions: TIS is crucial for the functional recovery of TASDH patients who underwent surgery.

\section{Background}

Traumatic acute subdural hematoma (TASDH) is one of the most devastating types of traumatic brain injury (TBI), with a mortality rate ranging from $30-70 \%[1,4,9,16]$. An emergent operation is considered if a patient is in coma or meets the surgical indication for TASDH. In 1981, Seelig et al. (1981) have reported that the mortality rate of TASDH can be reduced from $90-30 \%$ if the subdural hematoma was removed within 4 hours after injury [15]. Although few reports have shown similar findings [5, 18], a number of subsequent studies have failed to identify the effect of time to surgery on mortality rate $[3,8,9$, $14,19-22]$. In fact, some studies have reported a significant association between faster time to surgery and higher mortality rate $[2,17]$. Thus, we evaluated the data of TASDH patients who were surgically treated from 2008 to 2015 in Chia-Yi Christian Hospital in Taiwan. In this study, the effect of time from injury to surgery (TIS) on the outcomes of TASDH patients who were in coma from the time of trauma and who did not regain consciousness before surgical intervention was examined.

\section{Methods}

This study (CYCH-IRB 106074) was conducted after obtaining approval from the ethics committee of Chia-Yi Christian Hospital and has been performed in accordance with the ethical standards laid down in the 1964 Declaration of Helsinki and its later amendments. Patients with closed-head injury who had 
acute subdural hematoma on brain CT scan and who underwent craniotomy or craniectomy for the removal of hematoma were included in our study. However, patients with epidural hematoma, penetrating head injury, or intraparenchymal hemorrhage were excluded. Between January 2008 and December 2015, a total of 235 patients from the Neurosurgical Department of Chia-Yi Christian Hospital in Taiwan met the criteria for TASDH. Patients who had thoracic, abdominal, or pelvic injury $(n=10)$ or those who did not have any record of time of injury $(n=6)$ were excluded. Based on our exclusion criteria, patients with a coma scale score $>8(n=114$ patients, coma scale score of $9-15)$ and those aged $>70$ years $(n=19)$ were not included. Furthermore, 16 patients with a coma scale score of 3 or 4 and those with bilateral pupil dilation were excluded, of which 13 died and three were in vegetative state. A total of 70 patients met the criteria and were included for further analysis. In this study, surgical indication and treatment of acute subdural hematoma were in accordance with the guidelines on Surgical Management of Acute Subdural Hematoma [1]. In patients who were included in the study, the following data were extracted from the medical database of our hospital: age, sex, trauma mechanism, coma scale score, pupil size, and light reflex, midline shift on brain CT scan, whether craniotomy or craniectomy and evacuation of acute subdural hematoma were performed, postoperative intracranial pressure (ICP) in the surgical intensive care unit (mean data obtained during the second day after operation), information about postoperative complications or reoperation, time of injury notification (according to ambulance station record), arrival time at the emergency room of our hospital, time of surgery initiation, and surgical outcomes. TIS was defined as the documented time of injury notification to the initiation of surgery. The surgical outcomes were assessed using the Glasgow outcome scale (GOS) at least 1 year after the injury. These assessments were performed via phone calls to either the patients themselves or their closest relatives. In numerous cases, the outcomes were recorded during follow-up visit to the neurosurgeon. Functional recovery was defined as a GOS score of 4 or 5 . Severe neurological deficits, vegetative state, and death were considered poor outcomes.

\section{Statistical analysis}

The student's $t$-test or chi-square test was used for the comparison of variables in the functional and poor recovery groups (Table 1). In particular, student's $t$-test was utilized to evaluate continuous or numeric variables (such as age, coma scale, and midline shift on brain CT scan), and the chi-square test was used to assess non-numeric variables (including sex, traumatic mechanism, and type of operation). In addition, univariate and multivariate logistic regression models were used to analyze the impact of each variable in outcomes. All statistical analyses were performed using the Statistical Package for the Social Sciences software for Windows version 21.0 (IBM Corp., Armonk, NY, USA).

Age and coma scale were classified into three groups for analysis (Tables 1 and 2). P-values $<0.05$ were considered statistically significant.

Finally, the receiver operating characteristic curve was used for the analysis of TIS in the functional and poor recovery groups (Fig. 1). 


\section{Results}

Of the 235 TASDH patients who were surgically treated, 70 were included in our study. The demographic data of these patients are presented in Table 1. In total, 37 patients achieved functional recovery $(n=11$, GOS score of $5 ; n=26$, GOS score of 4 ) and 33 patients had poor recovery $(n=15$, severe neurological deficit; $n=9$, vegetative state; $n=9$, death). The mean age and standard deviation of the whole group was $50.9 \pm 14.6$ (range: $16-70$ ) years. Among the patients, 49 (70.0\%) were men and 21 (30\%) women. The mean coma scale score was $5.9 \pm 1.1$, the mean midline shift on brain CT scan was $10.0 \pm 5.2$, and the mean TIS was $162.5 \pm 45.6$ minutes (Table 1 ).

\section{Difference in each variable between the functional and poor recovery groups}

Age, sex, coma scale, trauma mechanism, pupil size and light reflex, type of operation (craniectomy or craniotomy), and midline shift on brain CT scan did not significantly differ between the two recovery groups (Table 1).

The postoperative ICP was significantly lower in the functional recovery group than in the poor recovery group $(p=0.003$, t-test). The TIS was $145.5 \pm 27.0$ minutes in the functional recovery group and $181.9 \pm$ 54.5 minutes in the poor recovery group. The student's t-test revealed that TIS is the most significant variable in distinguishing the two recovery groups $(p=0.001$, Table 1$)$.

\section{Analysis of variables in the univariate and multivariate logistic regression models}

Each variable (age, sex, coma scale, pupil size and light reflex, traumatic mechanism, type of operation, TIS, and midline shift on brain CT scan) was analyzed using the univariate and multivariate logistic regression models. The results revealed that TIS was a significant factor for functional outcomes in both regression models, and midline shift $\geq 10 \mathrm{~mm}$ on brain CT scan was a significant factor in the multivariate logistic regression model. Age, sex, coma scale, pupil size, type of operation, and traumatic mechanism were not significantly associated with functional outcomes (Table 2).

\section{Significance of TIS}

The TIS was analyzed with the ROC curve, and the results are shown in Fig. 1. The threshold time for functional recovery was 2 hours and 57.5 minutes. The specificity and sensitivity were 0.919 and 0.515 , respectively. This result indicated that the probability of functional recovery in a comatose TASDH patient who undergoes surgery within less than 2 hours and 57.5 minutes was $51.5 \%$ and that of a patient who undergoes surgery after the threshold time was $8.1 \%(100 \%-91.9 \%)$. The area under the curve was 0.713 , which indicated the credibility of the ROC curve, and the P-value was 0.002 .

\section{Discussion}


The present study aimed to determine whether TIS affects the degree of functional recovery in TASDH patients who required emergency craniotomy and removal of acute subdural hematoma. Various statistics were used in this study. Between the functional and poor recovery groups, only TIS showed a significant difference (Table 1). When a multivariate logistic regression model was applied, TIS and midline shift $\geq 10 \mathrm{~mm}$ on brain CT scan had a significant effect on functional recovery (Table 2). TIS was further analyzed with the ROC curve. To greatly improve the chance of achieving good functional recovery, the analysis result revealed that TIS should be within 2 hours and 57.5 minutes.

In previous studies, such as that of Dent et al. (1995), counter-intuitive results have shown that a shorter TIS is correlated to poor functional recovery $[2,17]$. However, such event is attributed to the existence of significant selection bias because patients with more severe injuries are more likely to undergo earlier surgery. This selection bias will certainly skew the results. Dent et al. (1995) have shown that patients who had surgery within 4 hours were more likely to have a lower Glasgow coma scale score, more severe intracranial injuries, and greater incidence of brain herniation than those who had surgery after 4 hours. To prevent a similar bias, only patients who had a coma scale score of 3-8, those younger than 70 years, and those who did not have additional structural brain injury other than TASDH were included. Moreover, patients with torso injuries were excluded as such conditions are commonly accompanied with hypotension and additional systemic complication. A multivariate logistic regression analysis that includes multiple variables will significantly reduce the likelihood of selection bias.

In the hypothesis of Mathai et al. (2010), the onset of life-threatening brain swelling in patients with severe TBI occurs between 2-3 hours after the injury and may be attributed to the osmotic load exerted by the breakdown of debris in the membrane and cytoplasmic structures [13]. In the report of Haselsberger et al. (1988), the surgical outcomes of TASDH patients were influenced by preoperative consciousness status [5]. When the time interval between onset of coma and surgical decompression exceeded 2 hours, the mortality rate increases from $47-80 \%$. Meanwhile, Seelig et al. (1981) have reported an increase in mortality rate from $30-90 \%$ if TIS exceeds 4 hours [15]. Our study is similar to the previous study; however, the current study focused on the functional outcomes of TASDH patients who were in coma and required emergency surgical operation. Our statistical analyses revealed that TIS was a significant factor and that the threshold time for surgery on TASDH patients must be assessed to achieve functional recovery.

With regard to the factors influencing outcomes, the impact of age and coma scale on functional recovery have been studied most frequently in the past $[6,7,12]$. Our data did not show that younger patients or those with a higher coma scale score were more likely to obtain better outcomes (Table 1), and this result may be attributed to two reasons. First, only 70 sets of data were included in our study, which may be considered a small sample size. Second, based on our exclusion criteria, 19 patients who were older than 70 years $(n=8$, severe neurological deficit; $n=7$, vegetative state; $n=4$, dead) and 16 patients with a coma scale score of 3 or 4 with bilateral pupil dilatation $(n=3$, vegetative state; $n=13$, dead) were not included. Thus, age and coma scale score were considered not significant factors. 
According to our multivariate logistic regression analysis, midline shift $\geq 10 \mathrm{~mm}$ on brain CT scan was another significant variable (Table 2). A patient with midline shift $\geq 5 \mathrm{~mm}$ on brain CT scan will experience prominent elevated ICP requiring surgical intervention [1]. In our study, we hypothesized that midline shift $\geq 10 \mathrm{~mm}$ on brain CT scan is associated with poor outcomes. The Rotterdam CT score is another tool that can be used to predict long-term outcomes via brain CT scan [10,11]. A higher Rotterdam score indicated a more severe brain injury, which is correlated to poor outcomes. To the best of our knowledge, this report first showed that midline shift $\geq 10 \mathrm{~mm}$ on brain CT scan was associated with poor outcomes.

As with all studies, the present study had some limitations. It had a small sample size and was conducted at a single center. Some exclusion criteria were also applied to age and coma scale score. Nevertheless, this study can be helpful in understanding the importance of TIS in patients with TBI and can provide valuable contributions in future-related studies.

The time lapse from injury was considered a critical factor based on the study of Seelig et al. (1981) in 1981. However, several authors have obtained different conclusions $[8,9,11,17,21,22]$. Our study included TASDH patients who were surgically treated from 2008 to 2015. With the use of the exclusion criteria, we believe that our sample is reasonable and that some obvious selection biases were eliminated. Thus, TIS is an important factor for the functional recovery of TASDH patients. Midline shift $\geq 10 \mathrm{~mm}$ on brain CT scan is another important factor associated with poor recovery.

\section{Conclusion}

TIS is crucial for the functional recovery of TASDH patients who underwent surgery. The threshold time for functional recovery of surgically treated TASDH patients were 2 hours and 57.5 minutes in our study.

\section{Declarations}

\section{Ethics approval and consent to participate:}

The study (CYCH-IRB 106074) was approved by the ethics committee at Chia-Yi Christian Hospital on September 25, 2017 and it is in accordance with the ethical standards laid down in the 1964 Declaration of Helsinki and its later amendments. For this is a retrospective study, informed consent is not required. Details that might disclose the identity of the subjects under study are omitted.

\section{Consent for publication}

I give my consent for information about my manuscript to be published in BMC neurology.

\section{Availability of data and materials}

The materials described in my manuscript will be freely available to any scientist who wish to use them for non-commercial purpose. 


\section{Competing Interests:}

This study does not have a financial relationship with the organization that sponsored the research: The authors declare no conflict of interest.

\section{Funding:}

No funding was received for this research.

\section{Author contribution}

Shih-Han Chen made contribution in the collection, statistical analysis of data and manuscript writing. Jui-Ming Sun and Wen-Kuei Fang contributed in acquisition of data and study concept.

\section{Acknowledgments:}

Special thanks from all of the co-authors of this essay to enago.tw for their provision of professional English reviewing service.

\section{Abbreviations}

CT: Computed tomography; GOS: Glasgow outcome scale; ROC: Receiver operating characteristic; TASDH: Traumatic acute subdural hematoma; TBI: Traumatic brain injury; TIS: Time from injury to surgery

\section{References}

1. Bullock MR, Chesnut R, Ghajar J, Gordon D, Hartl R, Newell DW, et al. Surgical management of acute subdural hematomas. Neurosurgery. 2006;58:S16-24.

2. Dent DL, Croce MA, Menke PG, Young BH, Hinson MS, Kudsk KA, et al. Prognostic factors after acute subdural hematoma. J Trauma. 1995;39:36-42.

3. Fountain DM, Kolias AG, Lecky FE, Bouamra O, Lawrence T, Adams H, et al. Survival trends after surgery for acute subdural hematoma in adults over a 20-year period. Ann Surg. 2017;265:590.

4. Gennarelli TA, Spielman GM, Langfitt TW, Gildenberg PL, Harrington T, Jane JA, et al. Influence of the type of intracranial lesion on outcome from severe head injury. J Neurosurg. 1982;56:26-32.

5. Haselsberger K, Pucher R, Auer L. Prognosis after acute subdural or epidural haemorrhage. Acta Neurochir. 1988;90:111-6.

6. Howard MA, 3rd, Gross AS, Dacey RG, Jr., Winn HR. Acute subdural hematomas: an age-dependent clinical entity. J Neurosurg. 1989;71:858-63.

7. Hukkelhoven CW, Steyerberg EW, Rampen AJ, Farace E, Habbema JD, Marshall LF, et al. Patient age and outcome following severe traumatic brain injury: an analysis of 5600 patients. J Neurosurg. 2003;99:666-73. 
8. Koç RK, Akdemir H, Öktem IS, Meral M, Menkü A. Acute subdural hematoma: outcome and outcome prediction. Neurosurg Rev. 1997;20:239-44.

9. Kotwica Z, Brzezinski J. Acute subdural haematoma in adults: an analysis of outcome in comatose patients. Acta Neurochir. 1993;121:95-9.

10. Langfitt TW, Gennarelli TA. Can the outcome from head injury be improved? J Neurosurg. 1982;56:19-25.

11. Leitgeb J, Mauritz W, Brazinova A, Janciak I, Majdan M, Wilbacher I, et al. Outcome after severe brain trauma due to acute subdural hematoma. J Neurosurg. 2012;117:324-33.

12. Marmarou A, Lu J, Butcher I, McHugh GS, Murray GD, Steyerberg EW, et al. Prognostic value of the Glasgow Coma Scale and pupil reactivity in traumatic brain injury assessed pre-hospital and on enrollment: an IMPACT analysis. J Neurotrauma. 2007;24:270-80.

13. Mathai KI, Sudumbrekar S, Sengupta S, Rappai T, Shashivadhanan M. Decompressive craniectomy in traumatic brain injury rationale and practice. Indian J Neurotrauma. 2010;7:9-12.

14. Park JH, Park JE, Kim SH, Lim YC, You NK, Ahn YH, et al. Outcomes of ultra-early decompressive craniectomy after severe traumatic brain injury-treatment outcomes after severe TBI. Korean $\mathrm{J}$ Neurotrauma. 2014;10:112-8.

15. Seelig JM, Becker DP, Miller JD, Greenberg RP, Ward JD, Choi SC. Traumatic acute subdural hematoma: major mortality reduction in comatose patients treated within four hours. $\mathrm{N}$ Engl $\mathrm{J}$ Med. 1981;304:1511-8.

16. Servadei F, Nasi MT, Cremonini AM, Giuliani G, Cenni P, Nanni A. Importance of a reliable admission Glasgow Coma Scale score for determining the need for evacuation of posttraumatic subdural hematomas: a prospective study of 65 patients. J Trauma. 1998;44:868-73.

17. Stone JL, Rifai MH, Sugar O, Lang RG, Oldershaw JB, Moody RA. Subdural hematomas. I. Acute subdural hematoma: progress in definition, clinical pathology, and therapy. Surg Neurol. 1983;19:216-31.

18. Tian HL, Chen SW, Xu T, Hu J, Rong BY, Wang G, et al. Risk factors related to hospital mortality in patients with isolated traumatic acute subdural haematoma: analysis of 308 patients undergone surgery. Chin Med J. 2008;121:1080-4.

19. Tien HC, Jung V, Pinto R, Mainprize T, Scales DC, Rizoli SB. Reducing time-to-treatment decreases mortality of trauma patients with acute subdural hematoma. Ann Surg. 2011;253:1178-83.

20. Walcott BP, Khanna A, Kwon CS, Phillips HW, Nahed BV, Coumans JV. Time interval to surgery and outcomes following the surgical treatment of acute traumatic subdural hematoma. J Clin Neurosci. 2014;21:2107-11.

21. Wilberger JE, Harris M, Diamond DL. Acute subdural hematoma: morbidity, mortality, and operative timing. J Neurosurg. 1991;74:212-8.

22. Wilberger JE, Jr., Harris M, Diamond DL. Acute subdural hematoma: morbidity and mortality related to timing of operative intervention. J Trauma. 1990;30:733-6. 


\section{Tables}

Table 1. Baseline demographic of the functional and poor recovery groups with traumatic acute subdural hematoma 


\begin{tabular}{|c|c|c|c|c|}
\hline & & \multicolumn{2}{|c|}{ Surgical outcomes } & \multirow[b]{2}{*}{$\begin{array}{c}\mathrm{P}- \\
\text { value }\end{array}$} \\
\hline & & $\begin{array}{l}\text { Functional recovery } \\
\qquad \begin{array}{l}\text { group } \\
\mathrm{n}=37\end{array}\end{array}$ & $\begin{array}{l}\text { Poor recovery } \\
\qquad \begin{array}{l}\text { group } \\
\mathrm{n}=33\end{array}\end{array}$ & \\
\hline \multirow[t]{4}{*}{ Age (mean $\pm S D$ ), years } & $50.9 \pm 14.6$ & $48.7 \pm 13.8$ & $53.3 \pm 15.3$ & 0.19 \\
\hline & $16-40$ & $10(27.03 \%)$ & $6(18.18 \%)$ & \\
\hline & $41-60$ & 18 (48.65\%) & $15(45.45 \%)$ & \\
\hline & $>60$ & $9(24.32 \%)$ & $12(36.36 \%)$ & \\
\hline \multirow[t]{3}{*}{ Sex } & & & & 0.638 \\
\hline & Male & $25(67.57)$ & $24(72.73)$ & \\
\hline & Female & $12(32.43)$ & $9(27.27)$ & \\
\hline \multirow[t]{3}{*}{ Cause of trauma } & & & & 0.729 \\
\hline & Traffic accidents & $25(67.57)$ & $21(63.64)$ & \\
\hline & Falls and others & $12(32.43)$ & $12(36.36)$ & \\
\hline \multirow[t]{4}{*}{ Coma scale (mean \pm SD) } & $5.9 \pm 1.1$ & $6.1 \pm 1.2$ & $5.8 \pm 1.1$ & 0.20 \\
\hline & 4 or 5 & $9(24.32)$ & $15(45.45)$ & \\
\hline & 6 & $17(45.95)$ & $11(33.33)$ & \\
\hline & 7 or 8 & $11(29.73)$ & $7(21.21)$ & \\
\hline \multirow[t]{5}{*}{ Pupil size } & & & & 0.306 \\
\hline & Normal & $21(56.76)$ & $14(42.42)$ & \\
\hline & Unilateral & $11(29.73)$ & $10(30.30)$ & \\
\hline & dilation & & & \\
\hline & Bilateral dilation & $5(13.51)$ & $9(27.27)$ & \\
\hline Midline shift (mean \pm SD) & $10.0 \pm 5.2 \mathrm{~mm}$ & $9.1 \pm 4.6$ & $10.9 \pm 5.8$ & 0.17 \\
\hline \multirow[t]{2}{*}{ on brain CT scan } & $<10 \mathrm{~mm}$ & $22(59.46)$ & $14(42.42)$ & \\
\hline & $\geq 10 \mathrm{~mm}$ & $15(40.54)$ & $19(57.58)$ & \\
\hline \multirow[t]{3}{*}{ ICP } & & & & 0.003 \\
\hline & $\leq 25$ & $35(94.59)$ & $22(66.67)$ & \\
\hline & $>25$ & $2(5.41)$ & 11 (33.33) & \\
\hline
\end{tabular}


Type of surgery

0.395

Craniectomy

Craniotomy

Injury to surgery (mins)
$21(56.76)$

$16(43.24)$

$145.5 \pm 27.0$
22 (66.67)

11 (33.33)

$181.9 \pm 54.5$

0.001

Table 2. Univariate and multivariate regression models 


\begin{tabular}{|c|c|c|c|c|}
\hline & $\begin{array}{l}\text { Crude OR } \\
(95 \% \mathrm{CI})\end{array}$ & P-value & $\begin{array}{l}\text { Adjusted OR } \\
\qquad(95 \% \text { CI })\end{array}$ & P-value \\
\hline \multicolumn{5}{|l|}{ Age } \\
\hline$\leq 40$ & 1 (reference) & & 1 (reference) & \\
\hline $41-60$ & 1.39 (0.41-4.72) & 0.598 & $1.22(0.2-7.52)$ & 0.829 \\
\hline$>60$ & $2.22(0.59-8.41)$ & 0.240 & $2.79(0.39-19.97)$ & 0.306 \\
\hline \multicolumn{5}{|l|}{ Sex } \\
\hline Male & 1 (reference) & & 1 (reference) & \\
\hline Female & $0.78(0.28-2.19)$ & 0.639 & $0.91(0.25-3.29)$ & 0.883 \\
\hline
\end{tabular}

Cause of trauma

Traffic accidents 1 (reference) 1 (reference)

Falls and others $1.19(0.44-3.20) \quad 0.730 \quad 0.50(0.12-2.1) \quad 0.342$

Coma scale

\begin{tabular}{ccccc}
\hline 4 or 5 & 1 (reference) & \multicolumn{3}{c}{1 (reference) } \\
\hline 6 & $0.39(0.13-1.19)$ & 0.098 & $0.5(0.07-3.57)$ & 0.489 \\
\hline 7 or 8 & $0.39(0.11-1.34)$ & 0.133 & $0.41(0.05-3.65)$ & 0.424
\end{tabular}

Pupil reaction

\begin{tabular}{ccccc}
\hline Isocoric & 1 (reference) & \multicolumn{3}{c}{1 (reference) } \\
\hline Unilateral dilation & $1.36(0.46-4.06)$ & 0.577 & $0.55(0.08-3.64)$ & 0.538 \\
\hline Bilateral dilation & $2.70(0.75-9.76)$ & 0.130 & $1.33(0.13-13.69)$ & 0.810 \\
\hline Midline shift & & & & \\
\hline$<10 \mathrm{~mm}$ & 1 (reference) & & 1 (reference) & \\
\hline$\geq 10 \mathrm{~mm}$ & $1.99(0.77-5.16)$ & 0.157 & $6.27(1.28-30.69)$ & 0.023 \\
\hline Type of surgery & & & & \\
\hline Craniectomy & 1 (reference) & & 1 (reference) & \\
\hline Craniotomy & $0.66(0.25-1.74)$ & 0.396 & $0.32(0.08-1.3)$ & 0.111 \\
\hline Time (TIS, mins) & $1.02(1.01-1.04)$ & 0.004 & $1.04(1.02-1.06)$ & 0.001 \\
\hline
\end{tabular}




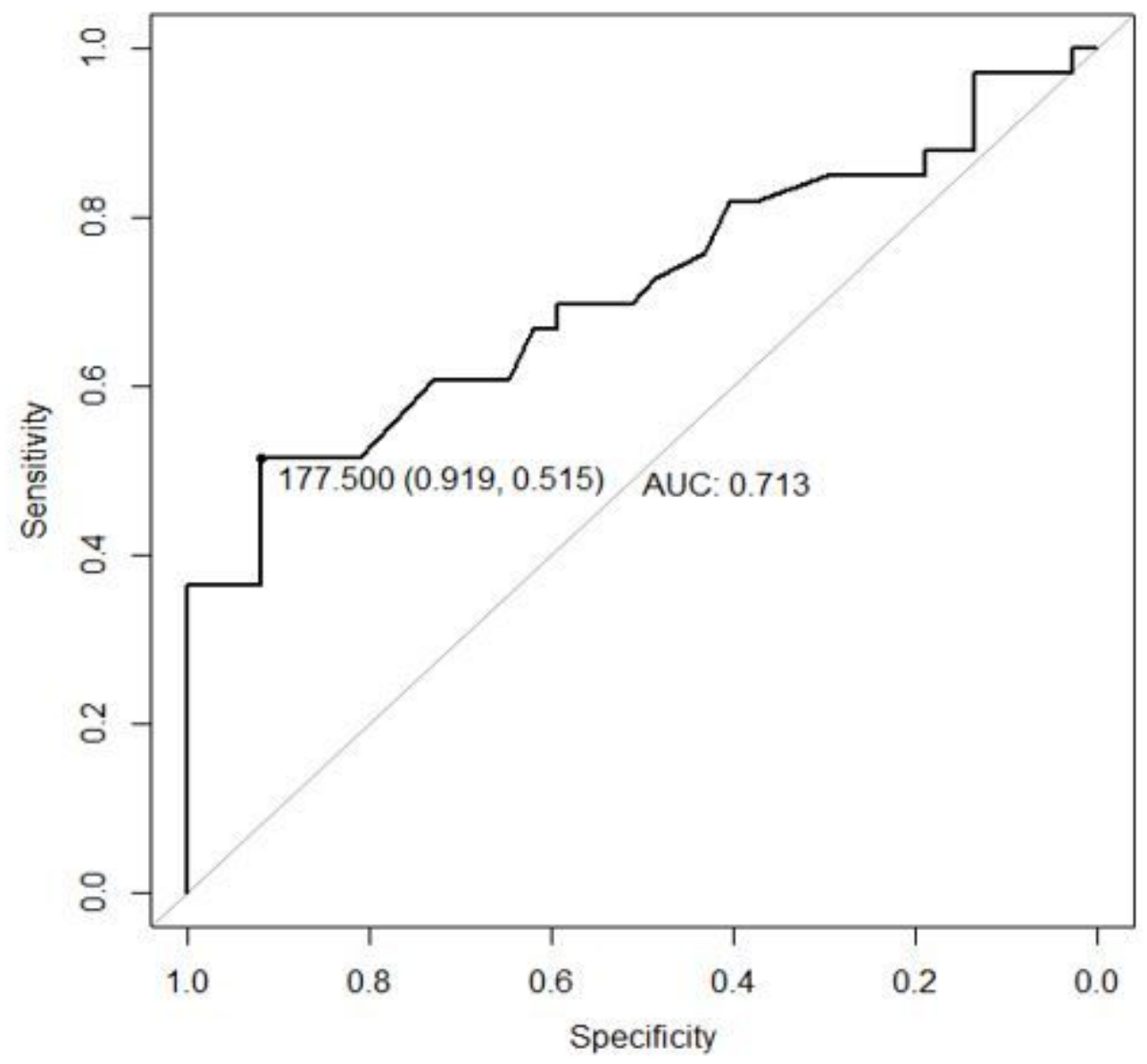

Figure 1

Time from injury to surgery ( $\mathrm{min}$ ) and surgical outcomes using the receiver operating characteristic curve. Area under the curve $=0.713$. Threshold time: $177.50 \mathrm{~min}(2 \mathrm{~h}$ and $57.5 \mathrm{~min})$. P-value $=0.002$, sensitivity $=0.515$, and specificity $=0.919$ 